\title{
The prognostic value of baseline hematological parameters of peripheral blood in metastatic gastric cancer treated with apatinib
}

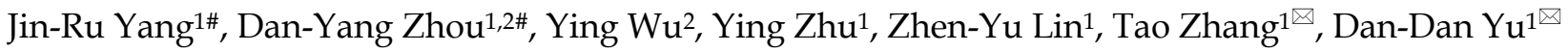 \\ 1. Cancer Center, Union Hospital, Tongii Medical College, Huazhong University of Science and Technology, Wuhan 430022, China; \\ 2. Department of Medical Oncology, Sun Yat-sen University Cancer Center, State Key Laboratory of Oncology in South China, Collaborative Innovation \\ Center for Cancer Medicine, 651 Dongfeng East Rd, Guangzhou, 510060, China. \\ \# These authors contributed equally to this article.
}

$\triangle$ Corresponding authors: Tao Zhang. Address: Cancer Center, Union Hospital, Tongji Medical College, Huazhong University of Science and Technology, Wuhan 430022, China. Tel: +86-27-85871982. Email: taozhangxh@hust.edu.cn. Dan-Dan Yu. Address: Cancer Center, Union Hospital, Tongji Medical College, Huazhong University of Science and Technology, Wuhan 430022, China. Tel: +86-27-85871982. Email: yudandan@hust.edu.cn.

(c) The author(s). This is an open access article distributed under the terms of the Creative Commons Attribution License (https://creativecommons.org/licenses/by/4.0/). See http:/ /ivyspring.com/terms for full terms and conditions.

Received: 2021.07.25; Accepted: 2021.11.01; Published: 2022.01.01

\begin{abstract}
Background: There is strong evidence that apatinib is effective in the treatment of third- or later-line advanced metastatic gastric cancer (mGC). Hematology prediction index is a convenient and cheap method to predict the prognosis of disease. However, the prognosis of baseline hematological parameters of peripheral blood, such as neutrophil-to-lymphocyte ratio (NLR), carbohydrate antigen 125 (CA125) and albumin (ALB) on mGC treated with apatinib have not been identified.

Methods: We retrospectively analyzed mGC received apatinib between 1 January 2014 and 30 June 2021. Survival analyses were performed using the Kaplan-Meier method and Cox-proportional hazards model.

Results: A total of 117 patients were included in this study. The cutoff value of NLR, CA125 and ALB was $2.25,19.24 \mathrm{U} / \mathrm{ml}$ and $37.60 \mathrm{~g} / \mathrm{L}$, respectively. The disease control rates (DCR) in the high and low NLR groups were $52.94 \%$ and $73.47 \%(P=0.024) ; 48.28 \%$ and $74.58 \%(P=0.003)$ in high and low CA125 groups; $72.97 \%$ and $41.86 \%(P=0.001)$ in high and low ALB groups. By survival analysis, increasing NLR $(P=0.003), C A 125(P<0.001)$ and decreasing ALB $(P<0.001)$ predicted a shorter PFS after apatinib. NLR $(P=0.015), C A 125(P=0.004)$ and ALB $(P=0.005)$ were significantly predictors for PFS in $\mathrm{mGC}$ treated with aptinib.
\end{abstract}

Conclusion: Increasing NLR, CA125 and decreasing ALB were associated with poorer clinical efficiency and prognosis after apatinib treatment.

Key words: Neutrophil-to-lymphocyte ratio; CA125; albumin; apatinib; metastatic gastric cancer

\section{Introduction}

Tumor angiogenesis is in an un-controlled and not-organized fashion, universally considered as an essential step in tumor growth and metastasis [1]. Vascular endothelial growth factor (VEGF) has been demonstrated to be the one which is majorly implicated in the pathological angiogenesis and is a signaling molecule that plays a role in several solid tumors [2]. Vascular endothelial growth factor receptor 2 (VEGFR-2) is one of the most potent angiogenic factors in the VEGF-induced angiogenic signaling [3]. Since gastric cancer (GC) express high VEGF and the 5-year survival of mGC with conventional chemotherapeutic agents is approximately $3.9 \%$ [4], targeting VEGF is therefore considered as an interesting therapeutic strategy for GC, especially metastatic gastric cancer (mGC). Apatinib, also known as YN968D1, inhibits VEGF-stimulated endothelial cell migration and 
proliferation and decreases tumor microvascular density through specifically binding VEGFR-2 [5]. As one of the latest orally antiangiogenic agents, apatinib makes encouraging preclinical and clinical achievements in a variety of solid tumors [6], including mGC [7]. It was also approved by China Food and Drug Administration (FDA) as a subsequent-line treatment for patients with $\mathrm{mGC}$ in 2014. However, finding predictive biomarkers that can discriminate patients who are most likely to be sensitive to apatinib and achieve personalized therapy is one of the challenges with antiangiogenic therapies using apatinib [8].

It is reported that inflammation had been validated as a high-risk factor for several cancers [9-12]. The cancer-generated inflammatory response results in upregulating cytokines and inflammatory mediators, inhibiting apoptosis, promoting angiogenesis, and damaging DNA and promoting the malignancy [9,10]. Neutrophil-to-lymphocyte ratio (NLR), as a mirror of systemic inflammatory response, has been considered as a useful indicator for predicting the prognosis and survival in various cancers [11], including GC [12]. Unfortunately, most of the previous studies focused on NLR as a prognostic factor in GC patients with operable early-stage [13] and metastatic disease with traditional chemotherapy [14]. Relevant studies have also shown that serum indexes such as tumor markers CA125 and ALB which can reflect body nutritional status, plays an important role in diagnosis GC $[15,16]$. However, the sensitivity of each indicator was low. It is unknown whether NLR, CA125 and ALB are suitable prognostic indicators for $\mathrm{mGC}$ patients receiving apatinib.

Based on this background, we aimed to evaluate whether NLR, CA125 and ALB can be used as a biomarker for predicting therapeutic response and survival outcome in $\mathrm{mGC}$ patients receiving apatinib.

\section{Methods}

\section{Patients}

We retrospectively analyzed 117 mGC patients diagnosed pathologically at cancer center, Union Hospital, Tongji Medical College, Huazhong University of Science and Technology between 1 January 2014 and 30 June 2021. Patients treated with regularly apatinib until disease progression or death in the study. Patients who had evidences of infection or hematological/autoimmune diseases, prior exposure to agents improving the number and function of blood cell, withdrawal of apatinib due to intolerable toxicity or death within 4 weeks from the first dose of apatinib therapy were excluded from the analysis. For all patients, baseline clinical-pathological characteristics, including age, gender, tumor sites, histology, Eastern Cooperative Oncology Group (ECOG) performance status, apatinib monotherapy or combination therapy and records of prior lines of chemotherapy were available for review. Baseline blood counts was defined as the results obtained within 7 days before initiating apatinib therapy. Reviewing data available at adjacent time points was to avoid selecting an outlier and ensure to record the representative outcomes. Tumor assessment was performed prior to first dose of apatinib therapy, at 2 circles of apatinib, and every 8-12 weeks thereafter, and clinical response were classified by imaging examinations of complete response (CR), partial response (PR), stable disease (SD), or progressive disease (PD) according to Response Evaluation Criteria in Solid Tumors (RECIST 1.1) [17]. Degrees of therapy-related adverse events (AEs) were graded by the Common Terminology Criteria for Adverse Events (CTCAE 4.0) [18]. All patients included were followed-up regularly until death or study data cutoff (30 June 2021). This retrospective study was approved by the Ethical Committees of Union Hospital, Tongji Medical College, Huazhong University of Science and Technology.

\section{Statistical analysis}

SPSS version 22.0 (SPSS Inc., Chicago, IL, USA) was used for statistical analyses. Baseline paraments of patients included were shown using Median (Quartile) $\left[M\left(P_{25}, P_{75}\right)\right]$. NLR was defined as the absolute neutrophil count divided by the absolute number of lymphocyte count. Analysis of receiver operating characteristic (ROC) curves was used to identify the cutoff value of variables. Median value severed as the cutoff value if area under the curve (AUC) of ROC was less than 0.50. Patient characteristics was compared by Chi-squared test and Student's t-test. Progression free survival (PFS) was determined from the date of the apatinib initiation to the date of progression as evidenced by radiographic assessment or obvious clinical manifestation, or death from any cause, or last follow-up (censored). Survival analyses were conducted using the Kaplan-Meier method and Cox-proportional hazards model. All tests were performed by two-sided, and $P<0.05$ were considered statistically significant.

\section{Results}

\section{Clinical characteristics}

A total of 117 Asian patients receiving apatinib for mGC between 1 January 2014 and 30 June 2021 were included in the retrospective study. Due to the intolerable toxicity, the patients discontinued were 
excluded according to CTCAE 4.0. The baseline characteristics of 117 patients were summarized in Table 1 . The median age was 53 (45-62) years old, including 45 (38.46\%) females and 72 (61.54\%) males. All patients received $250 \mathrm{mg} /$ day of apatinib. Sixty $(51.28 \%)$ patients received apatinib as a three-line or multi-line treatment. Moreover, $47(40.17 \%)$ patients received apatinib alone and 70 patients treated with combination of other chemotherapy agents. Followed up to 30 June 2021, the median PFS after apatinib was 2.33 months.

Table 1. General characteristics of $\mathrm{mGC}$ patients treated with apatinib $(\mathrm{N}=117)$

\begin{tabular}{ll}
\hline Variable & $\mathrm{n}(\%)$ \\
\hline Age (y) & $53(45-62)$ \\
Gender & $72(61.54 \%)$ \\
Male & $45(38.46 \%)$ \\
Female & \\
Tumor sites & $39(33.33 \%)$ \\
Antrum & $63(53.85 \%)$ \\
Non-antrum & $15(12.82 \%)$ \\
Unknown & \\
Histology & $65(55.56 \%)$ \\
Poor differentiation & $21(17.95 \%)$ \\
Non-poor differentiation & $31(26.50 \%)$ \\
Unknown & \\
ECOG & $104(88.89 \%)$ \\
$0-1$ & $13(11.11 \%)$ \\
2 & \\
Apatinib alone & $47(40.17 \%)$ \\
Yes & $70(59.83 \%)$ \\
No & \\
Lines of treatment & \\
$<3$ & $57(48.72 \%)$ \\
$\geq 3$ & $60(51.28 \%)$ \\
NLR & $2.64(1.56-4.53)$ \\
PLR & $170.86(111.60-236.51)$ \\
MLR & $0.36(0.26-0.53)$ \\
CA125 (U/ml) & $19.20(10.85-49.95)$ \\
CA199 (U/ml) & $17.00(5.09-309.80)$ \\
CEA (ug/L) & $5.04(2.08-17.75)$ \\
ALB (g/L) & $39.30(36.00-41.95)$ \\
GLB (g/L) & $27.30(24.40-30.50)$ \\
ALP (U/L) & $88.00(70.00-126.50)$ \\
Progress-free survival (m) & $2.33(1.42-4.10)$ \\
\hline
\end{tabular}

\section{Baseline NLR, CA125, ALB and treatment efficacy}

In the present study, the disease control rates (DCR) was $61.54 \%$ (72/117). Using ROC curves, the best cutoff for NLR, CA125, ALB were determined and the patients were divided into low NLR group $(\mathrm{NLR}<2.25)$ and high NLR group $(\mathrm{NLR} \geq 2.25)$; low CA125 group $($ CA125 $<3.50 \mathrm{U} / \mathrm{ml})$ and high CA125 group (CA125 $\geq 3.50 \mathrm{U} / \mathrm{ml})$; low ALB group (ALB $<37.60$ $\mathrm{g} / \mathrm{L}$ ) and high ALB group (ALB $\geq 37.60 \mathrm{~g} / \mathrm{L}$ ); DCR in the low and high NLR groups were $52.94 \%$ and $73.47 \%$ $(P=0.024) ; 48.28 \%$ and $74.58 \%(P=0.003)$ in high and low CA125 groups; $72.97 \%$ and $41.86 \%(P=0.001)$ in high and low ALB groups. No CR or PR was observed in both groups (Table 2A,B,C).

Table 2A. Effect of baseline NLR on clinical outcomes

\begin{tabular}{llllll}
\hline $\begin{array}{l}\text { Clinical } \\
\text { outcomes }\end{array}$ & $\begin{array}{l}\text { All } \\
(\mathrm{N}=117)\end{array}$ & $\begin{array}{l}\mathrm{NLR} \geq 2.25 \\
(\mathrm{~N}=68)\end{array}$ & $\begin{array}{l}\mathrm{NLR}<2.25 \\
(\mathrm{~N}=49)\end{array}$ & $\chi^{2}$ & $P$ \\
\hline Response rate & & & & & \\
SD & 72 & 36 & 36 & 5.070 & 0.024 \\
PD & 45 & 32 & 13 & & \\
PFS (months) & 2.33 & 1.83 & 3.50 & -2.341 & 0.021 \\
\hline
\end{tabular}

Table 2B. Effect of baseline CA125 on clinical outcomes

\begin{tabular}{llllll}
\hline $\begin{array}{l}\text { Clinical } \\
\text { outcomes }\end{array}$ & $\begin{array}{l}\text { All } \\
(\mathrm{N}=117)\end{array}$ & $\begin{array}{l}\text { CA125 } \geq 19.24 \\
\mathrm{U} / \mathrm{ml}(\mathrm{N}=58)\end{array}$ & $\begin{array}{l}\text { CA125<19.24 } \\
\mathrm{U} / \mathrm{ml}(\mathrm{N}=59)\end{array}$ & $x^{2}$ & $P$ \\
\hline Response rate & & & & & \\
SD & 72 & 28 & 44 & 8.548 & 0.003 \\
PD & 45 & 30 & 15 & & \\
PFS (months) & 2.33 & 1.72 & 3.33 & -3.288 & 0.002 \\
\hline
\end{tabular}

Table 2C. Effect of baseline ALB on clinical outcomes

\begin{tabular}{llllll}
\hline $\begin{array}{l}\text { Clinical } \\
\text { outcomes }\end{array}$ & $\begin{array}{l}\text { All } \\
(\mathrm{N}=117)\end{array}$ & $\begin{array}{l}\text { ALB } \geq 37.60 \\
\mathrm{~g} / \mathrm{L}(\mathrm{N}=74)\end{array}$ & $\begin{array}{l}\text { ALB<37.60 } \\
\mathrm{g} / \mathrm{L}(\mathrm{N}=43)\end{array}$ & $x^{2}$ & $P$ \\
\hline $\begin{array}{l}\text { Response rate } \\
\text { SD }\end{array}$ & 72 & 54 & 18 & 11.123 & 0.001 \\
PD & 45 & 20 & 25 & & \\
PFS (months) & 2.33 & 2.92 & 1.70 & 3.893 & 0.000 \\
\hline
\end{tabular}

\section{Baseline NLR, CA125, ALB and progression-free survival}

The elevated NLR, CA125 and reduced ALB was significantly correlated with poor prognosis for mGC patients treated with aptinib. The median PFS for patients with low NLR was 3.50 months, and for high NLR patients, it was 1.83 months $(P=0.024)$; PFS was 3.33 months in low CA125 group, and 1.72 months in high CA125 group ( $P=0.003) ; 1.70$ vs 2.92 months in low and high ALB group, respectively $(P=0.001)$. (Table 2A,B,C). By Kaplan-Meier analysis, increasing NLR $(P=0.003)$ and CA125 $(P<0.001)$ predicted a shorter PFS after apatinib in Figure 1,2; Figure 3 showed increasing ALB $(P<0.001)$ predicted a longer PFS after apatinib. Significant characteristics on univariate analysis, including gender (hazard ratio [HR] $=1.496,95 \%$ CI: 1.015-2.207, $P=0.042)$, NLR (HR=0.569, 95\% CI: 0.391-0.828, $P=0.003), \quad$ PLR (HR=0.576, 95\% CI: $0.360-0.921 ， P=0.021), \quad$ CA125 (HR=0.478, 95\% CI: 0.326-0.699, $P=0.000), \quad$ ALB (HR=2.160, 95\% CI: 1.446-3.225, $P=0.000)$ were carried into multivariate analysis. NLR ( $\mathrm{HR}=0.617,95 \% \mathrm{CI}$ : $0.419-0.910, \quad P=0.015), \quad$ CA125 $(\mathrm{HR}=0.560,95 \% \quad \mathrm{CI}$ : $0.377-0.831, P=0.004)$ and ALB $(\mathrm{HR}=1.873,95 \% \mathrm{CI}$ : 1.214-2.892, $P=0.005)$ were significantly associated with PFS by multivariate analyses (Table 3 ). 


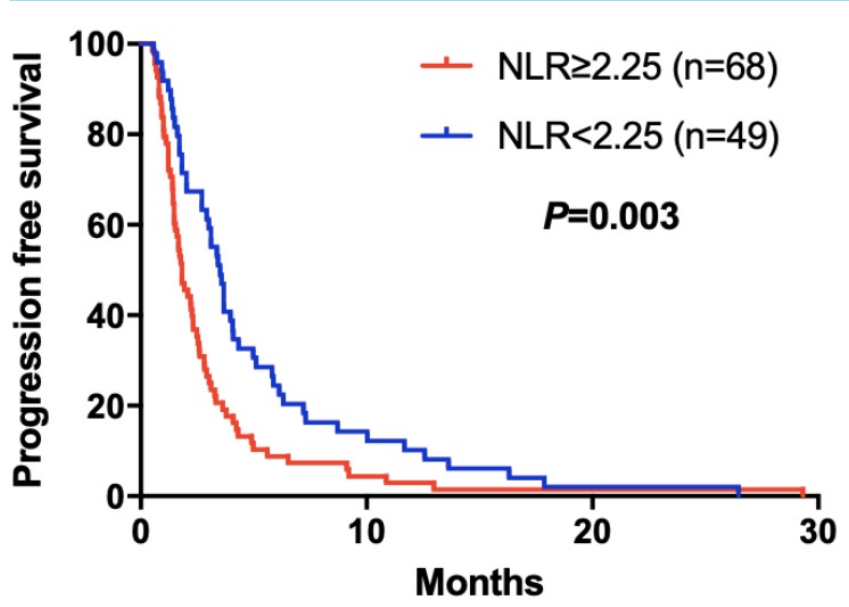

Figure 1. Kaplan-Meier curve for PFS of mGC patients with apatinib stratified by NLR

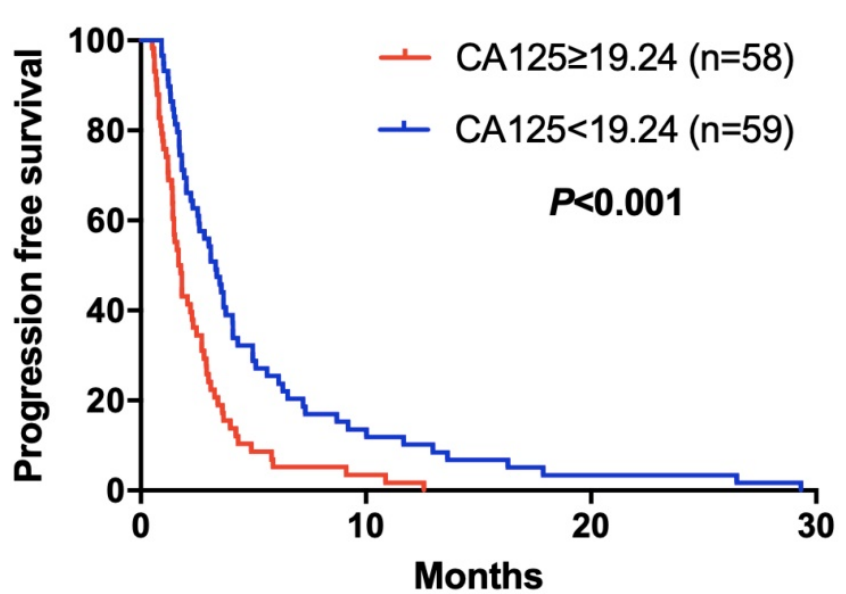

Figure 2. Kaplan-Meier curve for PFS of mGC patients with apatinib stratified by CA 125

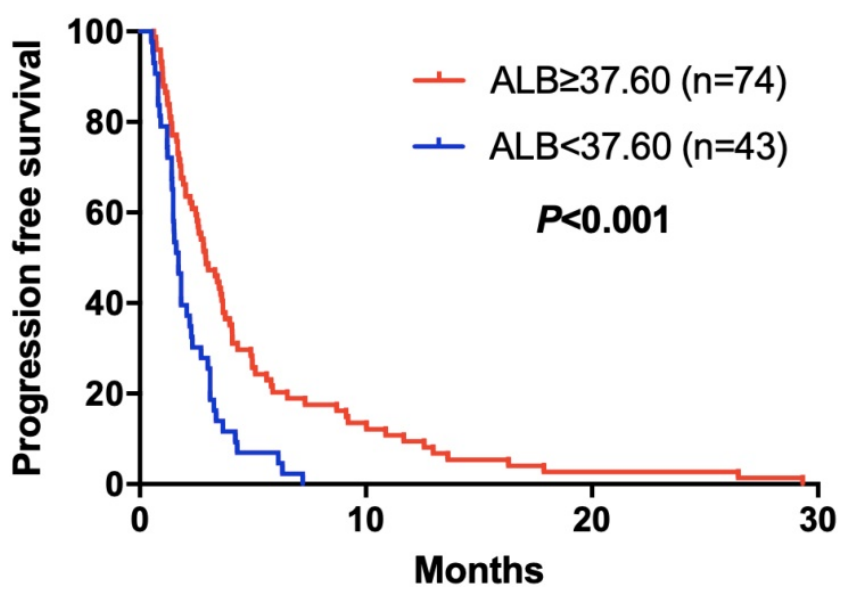

Figure 3. Kaplan-Meier curve for PFS of $\mathrm{mGC}$ patients with apatinib stratified by ALB

\section{Discussion}

As tumor angiogenesis is one of the hallmarks of malignancy, the inhibition of VEGF signaling has become an area of considerable interest regard to anticancer therapy. Apatinib, a novel receptor tyrosine kinase inhibitor selectively targeting
VEGFR-2, is considered as a promising therapeutic strategy across a range of cancers [6,19], including mGC $[8,20]$. Compared to other anti-angiogenic agents such as bevacizumab, sunitinib and sorafenib, apatinib has demonstrated a survival advantage in GC patients [21]. However, it is critically challenging to identify predictive biomarkers that could be used to select patients who will benefit most from current therapeutic strategies and avoid unnecessary cost as well as patients' discomfort.

Table 3. Cox proportional hazard regression analysis of progression-free survival in $\mathrm{mGC}$ patients treated with apatinib $(\mathrm{N}=117)$

\begin{tabular}{|c|c|c|c|c|}
\hline \multirow[t]{2}{*}{ Factors } & \multicolumn{2}{|l|}{ Univariable } & \multicolumn{2}{|l|}{ Multivariable } \\
\hline & HR $(95 \% \mathrm{CI})$ & $P$ & HR $(95 \%$ CI $)$ & $P$ \\
\hline Age $(\geq 53$ vs $<53)$ & $\begin{array}{l}1.340 \\
(0.927-1.935)\end{array}$ & 0.119 & & \\
\hline Gender (M vs F) & $\begin{array}{l}1.496 \\
(1.015-2.207)\end{array}$ & 0.042 & $\begin{array}{l}1.253 \\
(0.835-1.881)\end{array}$ & 0.276 \\
\hline $\begin{array}{l}\text { Tumor sites (Antrum vs } \\
\text { non-antrum) }\end{array}$ & $\begin{array}{l}1.303 \\
(0.977-1.737)\end{array}$ & 0.072 & & \\
\hline $\begin{array}{l}\text { Histology (Poor vs non-poor } \\
\text { differentiation) }\end{array}$ & $\begin{array}{l}1.110 \\
(0.888-1.386)\end{array}$ & 0.360 & & \\
\hline ECOG (0-1 vs 2$)$ & $\begin{array}{l}1.124 \\
(0.612-2.064)\end{array}$ & 0.707 & & \\
\hline Apatinib alone (Yes vs no) & $\begin{array}{l}0.994 \\
(0.682-1.448)\end{array}$ & 0.974 & & \\
\hline Lines of treatment $(\geq 3 \mathrm{vs}<3$ ) & $\begin{array}{l}0.983 \\
(0.678-1.426)\end{array}$ & 0.928 & & \\
\hline $\operatorname{NLR}(\geq 2.25$ vs $<2.25)$ & $\begin{array}{l}0.569 \\
(0.391-0.828)\end{array}$ & 0.003 & $\begin{array}{l}0.617 \\
(0.419-0.910)\end{array}$ & 0.015 \\
\hline $\operatorname{PLR}(\geq 257.61$ vs $<257.61)$ & $\begin{array}{l}0.576 \\
(0.360-0.921)\end{array}$ & 0.021 & $\begin{array}{l}0.787 \\
(0.473-1.309)\end{array}$ & 0.357 \\
\hline $\operatorname{MLR}(\geq 0.33$ vs $<0.33)$ & $\begin{array}{l}0.707 \\
(0.484-1.031)\end{array}$ & 0.072 & & \\
\hline $\begin{array}{l}\text { CA125 ( } \geq 19.24 \text { vs }<19.24 \\
\mathrm{U} / \mathrm{ml})\end{array}$ & $\begin{array}{l}0.478 \\
(0.326-0.699)\end{array}$ & 0.000 & $\begin{array}{l}0.560 \\
(0.377-0.831)\end{array}$ & 0.004 \\
\hline CA199 $(\geq 9.25$ vs $<9.25 \mathrm{U} / \mathrm{ml})$ & $\begin{array}{l}0.794 \\
(0.541-1.166)\end{array}$ & 0.239 & & \\
\hline CEA $(\geq 2.08$ vs $<2.08 \mathrm{ug} / \mathrm{L})$ & $\begin{array}{l}1.130 \\
(0.738-1.732)\end{array}$ & 0.573 & & \\
\hline $\operatorname{ALB}(\geq 37.60 \mathrm{vs}<37.60 \mathrm{~g} / \mathrm{L})$ & $\begin{array}{l}2.160 \\
(1.446-3.225)\end{array}$ & 0.000 & $\begin{array}{l}1.873 \\
(1.214-2.892)\end{array}$ & 0.005 \\
\hline GLB $(\geq 22.80$ vs $<22.80 \mathrm{~g} / \mathrm{L})$ & $\begin{array}{l}0.906 \\
(0.516-1.591)\end{array}$ & 0.732 & & \\
\hline $\operatorname{ALP}(\geq 65.50$ vs $<65.50 \mathrm{U} / \mathrm{L})$ & $\begin{array}{l}0.787 \\
(0.481-1.290)\end{array}$ & 0.342 & & \\
\hline
\end{tabular}

In this study, we evaluated the prognostic value of NLR, CA125 and ALB in mGC patients with apatinib. NLR, CA125 and ALB as timesaving, inexpensive and valid prognostic factors, were independently predictive factors for $\mathrm{mGC}$ treated with apatinib. Elevated NLR, CA125 and reduced ALB indicated poorer clinical response and survival for these patients.

Reported previously, occurrence of adverse events might act as surrogate biomarkers of drug activity [22], such as hypertension, hand-foot syndrome, and proteinuria [23], during antiangiogenic therapy. Treatment-emergent toxic effects, as a predictor of clinical outcomes, have their 
own limitations in addition to exposure to useless toxicity and high cost. Many marker assessment methods have limitations when it comes to reliability and reproducibility. Hypertension is a common and serious AEs regarding to apatinib. The inconsistent result among clinical trial, the recommended threshold, the time of occurrence and withdrawal or drug reduction causing difficulties in clinical application [22]. One of the advantages of baseline hematological indexes are available before the apatinib initiation compared to AEs, which can efficiently avoid the useless toxic medicine and high cost. Importantly, we further evaluate the best cutoff value of NLR (2.25), CA125 $(19.24 \mathrm{U} / \mathrm{ml})$ and ALB $(37.60 \mathrm{~g} / \mathrm{L})$ in mGC treated with apatinib. High NLR, CA125 and low ALB were significantly associated with worse response to therapy and shorter PFS. Interesting, patients in the lower NLR group appeared to demonstrate superior DCR compared to previous studies [24].

High NLR means a state of neutrophilia and/or lymphocytopenia. The tumor infiltrating neutrophils (TINs), as a component of neutrophils related to tumor, produce VEGF [25], cytokines as well as cytotoxic mediators. These factors promote the epithelial-mesenchymal transition and infiltration growth of tumor cells [26] and lead to tumor proliferation, angiogenesis, invasiveness and metastasis. Neutrophil, mast cells and other immune cells are not only a source of angiogenic/ lymphangiogenic molecules, but also their target [27]. Neutrophils are also found to sustain tumor revascularization in the context of anti-angiogenic therapy [28]. On the other hand, lymphocyte may limit tumor angiogenesis depending on the specific subtype and activation state and may induce the regression of tumor blood vessels in the context of immunotherapy [28]. Apatinib as an inhibitor targeting the vascular VEGF axis may exert their activity throughout the inhibition of VEGFR-2 phosphorylation, a principal mediator in the cancer angiogenic process [29].

CA125 is a tumor associated carbohydrate antigen, and its elevated serum level is related with many malignant tumors with low sensitivity [30]. Our study showed that the higher CA125 was, the shorter PFS and the lower DCR, which was consistent with the previously reported results.

Nutritional status in cancer patients is considered to be useful prognostic factors [31]. Malnutrition can further cause hormone imbalance, increase inflammatory cytokine infiltration, oxidative stress and so on [32]. ALB is an important serological indicator to evaluate human nutritional status. Low ALB level indicates poor nutritional status of the patient, which may lead to short PFS and DCR when treated with apatinib.

Of course, the results of this study had an inevitably deviation, because retrospective study design, and the small simple. To our knowledge, this study for the first time demonstrates a significant association between pretreatment hematological parameters of peripheral blood and apatinib therapy response in a cohort of mGC patients. This retrospective study was performed to preliminarily investigate the prognostic values of NLR, CA125 and ALB in $\mathrm{mGC}$ patients treated with apatinib, which is of great clinical value. Furthermore, this study provided theoretical and clinical evidence for further prospective studies.

\section{Conclusion}

Apatinib is a promising therapeutic strategy for mGC. However, it is critically challenging to identify predictive biomarkers. Baseline hematological parameters of peripheral blood (NLR, CA125 and ALB) as simple, inexpensive and readily available biomarkers, effetely predict response to apatinib and survival.

\section{Abbreviations}

ECOG: Eastern Cooperative Oncology Group performance status; NLR: neutrophil-to-lymphocyte ratio; PLR: platelet-to-lymphocyte ratio; MLR: monocyte-to-lymphocyte ratio; ALB: albumin; GLB: globulin; ALP: alkaline phosphatase; PFS: progression-free survival; SD: stable disease; PD: progressive disease.

\section{Acknowledgments}

This work was supported by the National Natural Science Foundation of China (No. 81872429).

\section{Ethical standards}

The Ethics Committee of the Union Hospital, Tongji Medical College, Huazhong University of Science and Technology granted approval for the study.

\section{Author Contributions}

(I) Conception and design: DD Yu and T Zhang; (II) Administrative support: DD Yu and T Zhang; (III) Provision of study materials or patients: ZY Lin; (IV) Collection and assembly of data: JR Yang, DY Zhou, Y $\mathrm{Wu}$ and $\mathrm{Y} \mathrm{Zhu}$; (V) Data analysis and interpretation: JR Yang and DY Zhou; (VI) Manuscript writing: All authors; (VII) Final approval of manuscript: All authors. 


\section{Competing Interests}

The authors have declared that no competing interest exists.

\section{References}

1. Kim TK, Park CS, Jang J, et al. Inhibition of VEGF-dependent angiogenesis and tumor angiogenesis by an optimized antibody targeting CLEC14a. Mol Oncol. 2018;12:356-72.

2. Ferrara N. VEGF and the quest for tumour angiogenesis factors. Nat Rev Cancer. 2002;2:795-803.

3. Peng FW, Liu DK, Zhang QW, et al. VEGFR-2 inhibitors and the therapeutic applications thereof: a patent review (2012-2016). Expert Opin Ther Pat. 2017;27:987-1004.

4. Wang J, Sun Y, Bertagnolli MM. Comparison of gastric cancer survival between Caucasian and Asian patients treated in the United States: results from the Surveillance Epidemiology and End Results (SEER) database. Ann Surg Oncol. 2015;22:2965-71.

5. Scott LJ. Apatinib: A Review in Advanced Gastric Cancer and Other Advanced Cancers. Drugs. 2018;78:747-58.

6. Lan CY, Wang Y, Xiong Y, et al. Apatinib combined with oral etoposide in patients with platinum-resistant or platinum-refractory ovarian cancer (AEROC): a phase 2, single-arm, prospective study. Lancet Oncol. 2018;19:1239-46.

7. Li J, Qin S, Xu J, et al. Randomized, Double-Blind, Placebo-Controlled Phase III Trial of Apatinib in Patients With Chemotherapy-Refractory Advanced or Metastatic Adenocarcinoma of the Stomach or Gastroesophageal Junction. J Clin Oncol. 2016;34:1448-54.

8. Roviello G, Ravelli A, Polom K, et al. Apatinib: A novel receptor tyrosine kinase inhibitor for the treatment of gastric cancer. Cancer Lett. 2016;372:187-91.

9. Coussens LM, Werb Z. Inflammation and cancer. Nature. 2002;420:860-7.

10. Greten FR, Grivennikov SI. Inflammation and Cancer: Triggers, Mechanisms, and Consequences. Immunity. 2019;51:27-41.

11. Mei Z, Shi L, Wang B, et al. Prognostic role of pretreatment blood neutrophil-to-lymphocyte ratio in advanced cancer survivors: A systematic review and meta-analysis of 66 cohort studies. Cancer Treat Rev. 2017:58:1-13.

12. Bowen RC, Little NAB, Harmer JR, et al. Neutrophil-to-lymphocyte ratio as prognostic indicator in gastrointestinal cancers: a systematic review and meta-analysis. Oncotarget. 2017;8:32171-89.

13. Wang SC, Chou JF, Strong VE, et al. Pretreatment Neutrophil to Lymphocyte Ratio Independently Predicts Disease-specific Survival in Resectable Gastroesophageal Junction and Gastric Adenocarcinoma. Ann Surg. 2016;263:292-7.

14. Zhou D, Wu Y, Zhu Y, et al. The Prognostic Value of Neutrophil-tolymphocyte Ratio and Monocyte-to-lymphocyte Ratio in Metastatic Gastric Cancer Treated with Systemic Chemotherapy. J Cancer. 2020;11:4205-12.

15. Feng F, Tian Y, Xu G, et al. Diagnostic and prognostic value of CEA, CA19-9, AFP and CA125 for early gastric cancer. BMC Cancer. 2017;17:737.

16. Abe A, Kurita K, Hayashi $\mathrm{H}$, et al. Correlation between prognostic nutritional index and occlusal status in gastric cancer. Oral Dis. 2020;26:465-72.

17. Wolchok JD, Hoos A, O'Day S, et al. Guidelines for the evaluation of immune therapy activity in solid tumors: immune-related response criteria. Clin Cancer Res. 2009;15:7412-20.

18. Dueck AC, Mendoza TR, Mitchell SA, et al. Validity and Reliability of the US National Cancer Institute's Patient-Reported Outcomes Version of the Common Terminology Criteria for Adverse Events (PRO-CTCAE). JAMA Oncol. 2015;1:1051-9.

19. Li L, Xiao S, Zhang L, et al. An open label, single-armed, exploratory study of apatinib (a novel VEGFR-2 tyrosine kinase inhibitor) in patients with relapsed or refractory non-Hodgkin lymphoma. Oncotarget. 2018;9:16213-9.

20. Zhao TT, Xu H, Xu HM, et al. The efficacy and safety of targeted therapy with or without chemotherapy in advanced gastric cancer treatment: a network meta-analysis of well-designed randomized controlled trials. Gastric Cancer. 2018;21:361-71.

21. Roviello G, Petrioli R, Marano L, et al. Angiogenesis inhibitors in gastric and gastroesophageal junction cancer. Gastric Cancer. 2016;19:31-41.

22. Fuchs CS, Tomasek J, Yong CJ, et al. Ramucirumab monotherapy for previously treated advanced gastric or gastro-oesophageal junction adenocarcinoma (REGARD): an international, randomised, multicentre, placebo-controlled, phase 3 trial. Lancet. 2014;383:31-9.

23. Hamnvik OP, Choueiri TK, Turchin A, et al. Clinical risk factors for the development of hypertension in patients treated with inhibitors of the VEGF signaling pathway. Cancer. 2015;121:311-9.

24. Chen J, Wang J. Efficacy and safety assessment of apatinib in patients with advanced gastric cancer: a meta-analysis. Onco Targets Ther. 2018;11:4149-58.

25. Strieter RM, Burdick MD, Mestas J, et al. Cancer CXC chemokine networks and tumour angiogenesis. Eur J Cancer. 2006:42:768-78.

26. Gaida MM, Steffen TG, Günther F, et al. Polymorphonuclear neutrophils promote dyshesion of tumor cells and elastase-mediated degradation of E-cadherin in pancreatic tumors. Eur J Immunol. 2012;42:3369-80.
27. Varricchi G, Loffredo S, Galdiero $M R$, et al. Innate effector cells in angiogenesis and lymphangiogenesis. Curr Opin Immunol. 2018;53:152-60.

28. De Palma M, Biziato D, Petrova TV. Microenvironmental regulation of tumour angiogenesis. Nat Rev Cancer. 2017;17:457-74.

29. Witte L, Hicklin DJ, Zhu Z, et al. Monoclonal antibodies targeting the VEGF receptor-2 (Flk1/KDR) as an anti-angiogenic therapeutic strategy. Cancer Metastasis Rev. 1998;17:155-61.

30. Huang C, Liu Z, Xiao L, et al. Clinical Significance of Serum CA125, CA19-9, CA72-4, and Fibrinogen-to-Lymphocyte Ratio in Gastric Cancer With Peritoneal Dissemination. Front Oncol. 2019;9:1159.

31. Sakurai K, Ohira M, Tamura T, et al. Predictive Potential of Preoperative Nutritional Status in Long-Term Outcome Projections for Patients with Gastric Cancer. Ann Surg Oncol. 2016;23:525-33.

32. Roxburgh C, Mcmillan DC. Cancer and systemic inflammation: treat the tumour and treat the host. Br J Cancer. 2014;110:1409-12. 